\title{
Application of the iterative probe correction technique for a high-order probe in spherical near-field antenna measurements
}

\author{
Laitinen, Tommi; Pivnenko, Sergey; Breinbjerg, Olav
}

Published in:

I E E E Antennas and Propagation Magazine

Link to article, DOI:

10.1109/MAP.2006.1715282

Publication date:

2006

Document Version

Publisher's PDF, also known as Version of record

Link back to DTU Orbit

Citation (APA):

Laitinen, T., Pivnenko, S., \& Breinbjerg, O. (2006). Application of the iterative probe correction technique for a high-order probe in spherical near-field antenna measurements. I E E E Antennas and Propagation Magazine, 48(4), 179-185. https://doi.org/10.1109/MAP.2006.1715282

\section{General rights}

Copyright and moral rights for the publications made accessible in the public portal are retained by the authors and/or other copyright owners and it is a condition of accessing publications that users recognise and abide by the legal requirements associated with these rights.

- Users may download and print one copy of any publication from the public portal for the purpose of private study or research.

- You may not further distribute the material or use it for any profit-making activity or commercial gain

- You may freely distribute the URL identifying the publication in the public portal 


\section{Technical Introduction}

This month"s highlighted AMTA work comes to us from the Technical University of Denmark, one of several European institutions that have studied various near-field antenna-measurement techniques over the years. This month, authors Tommi Laitinen, Sergey Pivnenko, and Olav Breinbjerg present a paper titled "Application of the Iterative Probe-Correction Technique for a High-Order Probe in Spherical Near-Field Antenna Measurements." Generally speaking, many papers have been written regarding probe correction for simple, "first-order" near-field probes, like open circular waveguide probes and a circular waveguide-fed conical hom. With some "stretch," the theory has even been applied to square waveguide probes and odd-order modes. For wideband measurements, one may wish to employ broadband probe antennas, such as a dual-ridged hom. Since simple first-order corrections don't work well for such antennas, the authors have developed a model that would allow the use of such classes of antennas for spherical near-field scanning. I am sure you will find the paper useful and informative.
The next time you hear from me in this column, AMTA 2006 will join the rest of the year in the can. Don't look back and say you wished you had attended! Register today. I hope to see you all in Austin. In the meantime, send constructive criticisms via e-mail to brian.kent@wpafb.af.mil. AP-S Magazine accolades, however, are solely reserved for the true force behind this magazine, r.stonc@ieee.org. Keep up the great work, Ross, and may the force continue to be with you. Until next time.

$\gamma$

\section{Reference}

1. Brian M. Kent, John Watkins, Kyle Freund1, Thomas Coveyou, Lisa Cravens, Alan Buterbaugh, Anthony D. Griffith, and Robert Scully, "Electromagnetic Interference Attenuation Test of the Space Shuttle Discovery Using the Air Force Research Laboratory Mobile Diagnostic Laboratory," IEEE Antennas and Propagation Magazine, 47, 6, December 2005, pp. 128-134.

\title{
Application of the Iterative Probe- Correction Technique for a High-Order Probe in Spherical Near-Field Antenna Measurements
}

\author{
Tommi Laitinen, Sergey Pivnenko, and Olav Breinbjerg \\ Electromagnetic Systems, Ørsted-DTU, Technical University of Denmark \\ DK-2800 Kgs. Lyngby, Denmark \\ E-mail: tla@oersted.dtu.dk
}

\begin{abstract}
An iterative probe-correction technique for spherical near-field antenna measurements is examined. This technique has previously been shown to be well-suited for non-ideal first-order probes. In this paper, its performance in the case of a highorder probe (a dual-ridged horn) is examined.
\end{abstract}

Keywords: Antenna measurements; near field measurements; antenna radiation pattern; probe antennas; probe correction; horn antennas 


\section{Introduction}

$\mathbf{P}$ robe-corrected spherical near-field antenna measurements constitute an accurate method for characterizing the radiation of antennas [1]. Typically, the probe-corrected spherical near-field antenna measurements are based on the use of a first-order $(\mu= \pm 1)$ probe and the application of the traditional first-order probe correction [1].

Examples of typically used first-order probes are an openended circular waveguide probe, and a conical horn fed by a circular waveguide, excited with the $T E_{11}$ waveguide mode [1]. Square and rectangular waveguide probes, although being odd-order probes [2], approximate first-order probes to such an extent that they are sometimes applied together with the first-order probe correction [3].

Due to the finite accuracy of manufacturing of probes, even nominally first-order probes are never exactly first-order probes. For this reason, the application of the traditional first-order probe correction technique, together with a real first-order probe, provides a small error in the predicted radiation pattern. If the probe has been manufactured well, the errors in the far-field prediction are typically negligible. For a poorly-manufactured first-order probe, the errors may be unacceptable. Similarly, using a square or a rectangular waveguide probe together with a first-order probecorrection technique may provide acceptable accuracy in some applications, whereas in other applications - in particular, e.g, in an off-axis measurement [4] - accurate far-field prediction will typically not be obtained.

A high-order $(|| \mu \mid>1)$ probe-correction technique can be applied to avoid the requirement for tight manufacturing tolerances of the probes and/or the requirement of using a first-order probe for accurate antenna-pattern characterization [5]. Compared to the first-order probe correction, a disadvantage of most known and practical high-order probe-correction techniques is their higher computational complexity.

A computationally efficient iterative probe-correction technique was presented recently in [6]. This technique is well-suited for non-ideal first-order probes. Furthermore, the examination reported in [5] showed that the iterative probe-correction technique can, in some cases, be used for other probes, too, e.g., for an openended rectangular waveguide probe, and thus not only for non-ideal first-order probes. Yet, the same examination has also revealed that the technique fails in some other cases.

The purpose of this paper is to show that the iterative probecorrection technique can be applied for probes other than non-ideal first-order probes. Some guidelines for the application range of the technique are given. These results were obtained by simulations of spherical near-field measurements of an AUT (antenna under test) model at several frequencies, using a probe model representing a dual-ridged horn.

In this paper, the theory related to the iterative probe-correction technique is first presented in Section 2. Second, the AUT and the probe models used in the simulations are presented in Section 3. Simulations and results are presented in Section 4. Finally, the conclusions are drawn in Section 5.

\subsection{Spherical-Wave Expansion}

In conventional probe-corrected spherical near-field antenna measurements, the fields radiated by both the AUT and the probe, outside their minimum spheres, are expressed by spherical-wave expansions. For the AUT, this is expressed as

$$
\bar{E}(r, \theta, \phi)=k \sqrt{\eta} \sum_{s=1}^{2} \sum_{n=1}^{N} \sum_{m=-n}^{n} Q_{s m n} \bar{F}_{s m n}^{(3)}(r, \theta, \phi),
$$

where $(r, \theta, \phi)$ are the standard spherical coordinates, $k$ is the wavenumber, $\eta$ is the intrinsic impedance of the medium, $N$ is the truncation number for the spherical-wave expansion, the $Q_{s m n}$ are the spherical-wave coefficients associated with the AUT field, and the $\bar{F}_{s m n}^{(3)}(r, \theta, \phi)$ are the spherical vector wave functions [1].

The spherical-wave expansion of Equation (1) also holds for the electric field of the probe by replacing the indices $(s, m, n)$ by $(\sigma, \mu, v), N$ by $\nu_{\max }$, and $Q_{s m n}$ by $Q_{\sigma \mu \nu}^{(p)}$. The formulation of the probe-corrected spherical near-field antenna measurements is based on the assumption of the known probe receiving coefficients, $R_{\sigma \mu \nu}^{(p)}$. For a reciprocal probe, these are related to $Q_{\sigma \mu \nu}^{(p)}$ as follows:

$$
R_{\sigma \mu \nu}^{(p)} \cong(-1)^{\mu} Q_{\sigma-\mu \nu}^{(p)}
$$

It is typically sufficient to truncate the spherical-wave series of the AUT at $N=\left[k r_{0}\right]+10$, where $r_{0}$ is the radius of the minimum sphere of the AUT, and a similar expression holds for the probe, as well.

\subsection{Transmission Formula}

The signal received by the probe at the position $(r, \theta, \phi)$ is expressed as a function of the spherical-wave coefficients of the AUT field and the probe receiving coefficients. This relation, the transmission formula, can be expressed as [1]

$$
w(r, \chi, \theta, \phi)=\sum_{s} \sum_{n} \sum_{m} Q_{s m n} \sum_{\mu} e^{i m \phi} d_{\mu m}^{n}(\theta) e^{i \mu \chi} P_{s \mu n}^{(p)}(k r)
$$

where $\chi$ is the probe rotation angle, $d_{\mu m}^{n}(\theta)$ is a rotation function defined in [1], and the probe response constants are

$$
P_{s \mu n}^{(p)}(k r)=\frac{1}{2} \sum_{\sigma} \sum_{v} C_{\sigma \mu v}^{s n(3)}(k r) R_{\sigma \mu v}^{(p)}
$$

where the $C_{\sigma \mu v}^{s n(3)}(k r)$ are the translation coefficients defined in [1]. The geometry related to the probe-corrected spherical near-field antenna measurements is presented in Figure 1. 


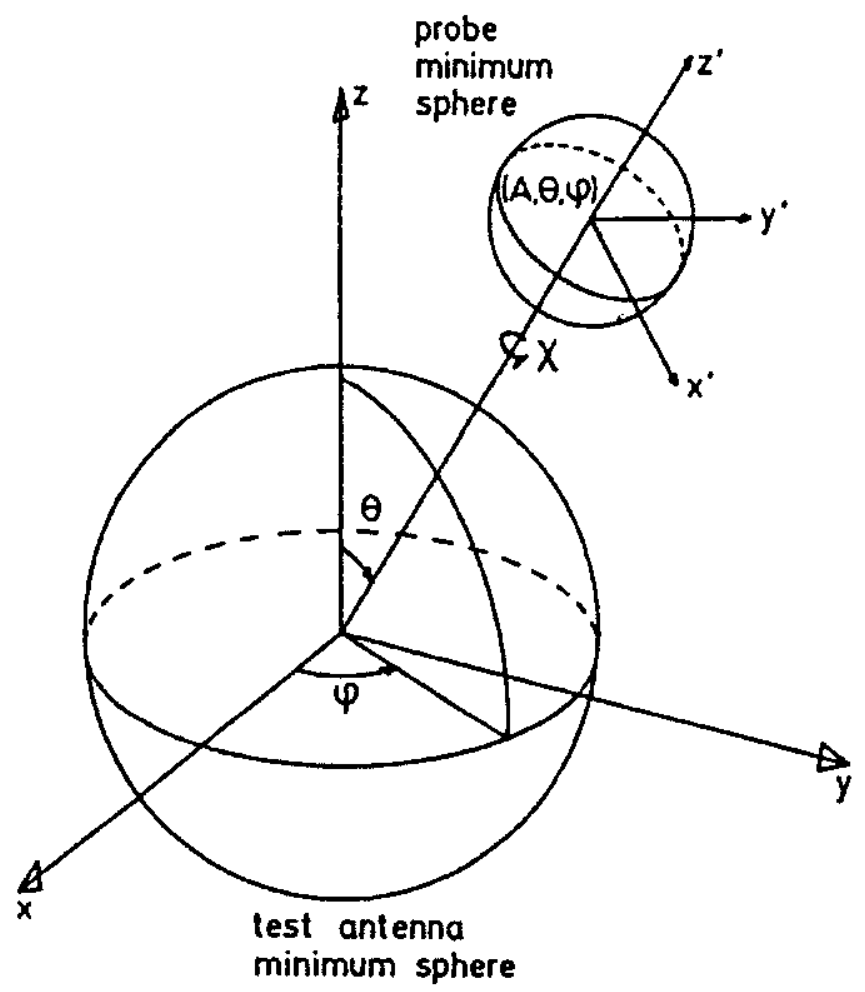

Figure 1. The geometry of the probe-corrected spherical nearfield antenna measurements. The $\left(x^{\prime}, y^{\prime}, z^{\prime}\right)$ refer to the probe coordinates.

\subsection{Iterative Probe-Correction Technique}

The iterative probe-correction technique presented in [6] is now briefly reviewed. First, the received signal in the transmission formula is written as a sum of the desired signal, $w_{d}(r, \chi, \theta, \phi)$, and the error signal, $w_{e}(r, \chi, \theta, \phi)$,

$$
w(r, \chi, \theta, \phi)=w_{d}(r, \chi, \theta, \phi)+w_{e}(r, \chi, \theta, \phi),
$$

where

$$
w_{d}(r, \chi, \theta, \phi)=\sum_{n=1}^{N} \sum_{m=-n}^{n} \sum_{s=1}^{2} \sum_{\mu= \pm 1} Q_{s m n} e^{i m \phi} d_{\mu m}^{n}(\theta) e^{i \mu \chi} P_{s \mu n}^{(p)}(k r)
$$

and

$$
w_{e}(r, \chi, \theta, \phi)=\sum_{n=1}^{N} \sum_{m=-n}^{n} \sum_{s=1}^{2} \sum_{\mu \neq \pm 1} Q_{s m n} e^{i m \phi} d_{\mu m}^{n}(\theta) e^{i \mu \lambda} P_{s \mu n}^{(p)}(k r)
$$

Note that the equations for the desired and error signals differ only in the $\mu$ summation. In case of an ideal first-order $(\mu= \pm 1)$ probe, the application of the traditional first-order probe-correction technique would provide a correct solution to the unknown $Q_{s m n}$ because the error signal, $w_{e}(r, \chi, \theta, \phi)$, would be zero. In practice, $w_{e}(r, \chi, \theta, \phi)$ in Equation (6) is always nonzero.
The iterative probe-correction technique [6] is based on the idea of first estimating the $Q_{s m n}$ of the AUT field sufficiently accurately using the traditional first-order probe-correction technique, i.e., by neglecting the zeroth and higher-order modes of the probe. Thus, in general, the smaller is the ratio between $w_{e}(r, \chi, \theta, \phi)$ and $w_{d}(r, \chi, \theta, \phi)$, the more accurate is the estimate of the $Q_{s m n}$ of the AUT. The $Q_{s m n}$ estimated this way are called the zeroth-round estimate of the $Q_{s m n}$ of the AUT $\left(Q_{s m n}^{(0)}\right)$.

The iterative technique consists of three steps. These three steps are illustrated in Figure 2, where the data flowchart of the iterative probe-correction technique is presented. First, the error signal, $w_{e}^{(1)}$, is estimated by calculating it from Equation (6) using $Q_{s m n}^{(0)}$. Second, the estimated error signal is subtracted from the original received signal, $w$. The signal thus obtained is called the first-round estimate of the desired signal, $w_{d}^{(1)}$. Third, the traditional first-order probe correction is applied to the first-round estimate of the desired signal to provide a first-round estimate of the $Q_{s m n}$ of the AUT ( $Q_{s m n}^{(1)}$ ). At this point, one iteration round has been accomplished. The second round starts by re-estimating the error signal by using Equation (6), but now by using $Q_{s m n}^{(1)}$.
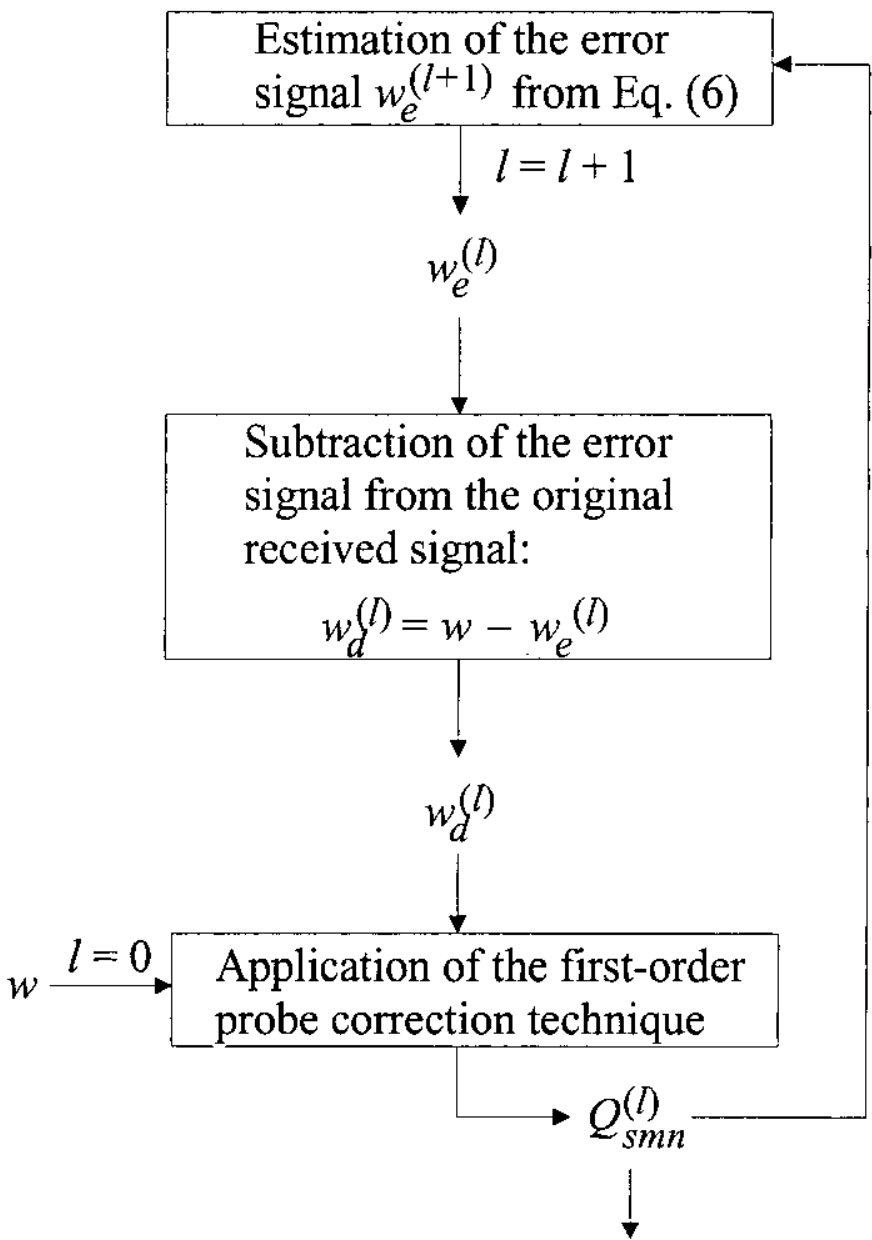

Figure 2. The data flowchart of the iterative probe-correction technique. 


\section{AUT and Probe Models}

The AUT and the probe models used in the simulation of this paper were represented by sets of spherical-wave coefficients of the AUT $\left(Q_{s m n}\right)$ and by scts of receiving coefficients of the probe $\left(R_{\sigma a V}^{(p)}\right.$ ), respectively. In the following, the AUT and probe models are described.

\subsection{AUT Model}

The AUT model was constructed from the antenna shown in Figure 3. This antenna consisted of nine Huygens sources separated by $\lambda / 2$. The center point of the Huygens source grid was located at an off-axis distance $d$ from the rotation center of the spherical measurement setup. The maximum radiation of each Huygens source was in the $+z$ axis direction. The electric field of each Huygens source was $y$ polarized, and in the same phase in the far field in the $+z$ axis direction. The radiation pattern of the antenna represented, e.g., an element pattern of a linear antenna array. The antenna, with various off-axis distances $d$, thus constituted a relatively realistic set of radiation patterns to be used in simulations.

A set of $Q_{s m n}$ for the AU'T model was determined by performing the spherical-wave expansion of the radiated field of the antenna. This was repeated for different off-axis distances, $d$, of the antenna. In this paper, the spherical-wave coefficients were determined up to the truncation number $N=\left[k r_{0}\right]+12$. It was noted that the field related to the $Q_{s m n}$ of the AUT model did not strictly correspond to the radiated field of the antenna shown in Figure 3, but it was a very accurate approximation of the radiated field of the antenna. This was mainly due to the truncation errors in performing the spherical-wave expansion.

In Figure 4, the co-polar directivities of the AUT model, calculated according to Ludvig's third definition [7], are presented for the $\phi=0^{\circ}, 45^{\circ}$, and $90^{\circ}$ planes, with the reference angle $\phi_{0}=90^{\circ}$. The peak co-polar directivity of the AUT model was approximately $14.2 \mathrm{dBi}$.

It was noted that the directivities of the AUT model remained the same regardless of the off-axis distance $d$. However, this did not mean that the radiation patterns of the antenna with various off-

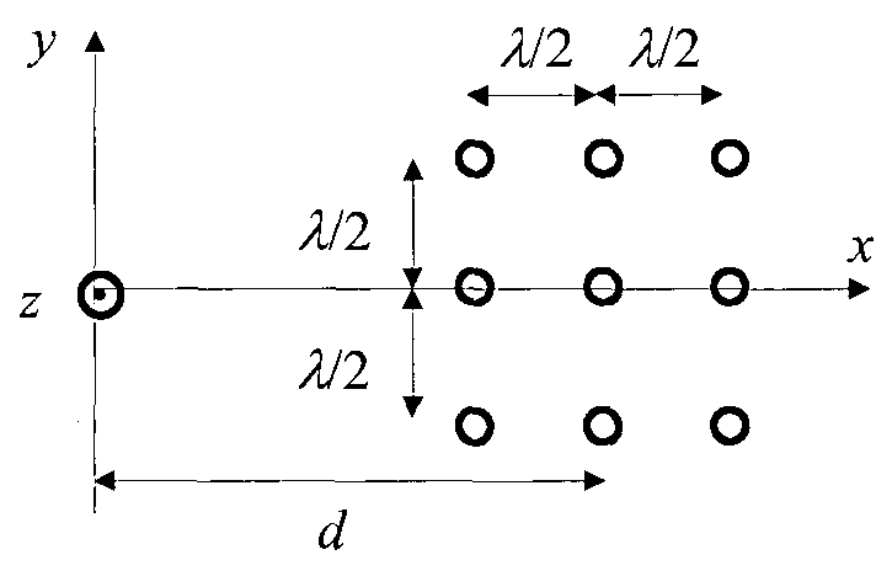

Figure 3. The AUT antenna model.

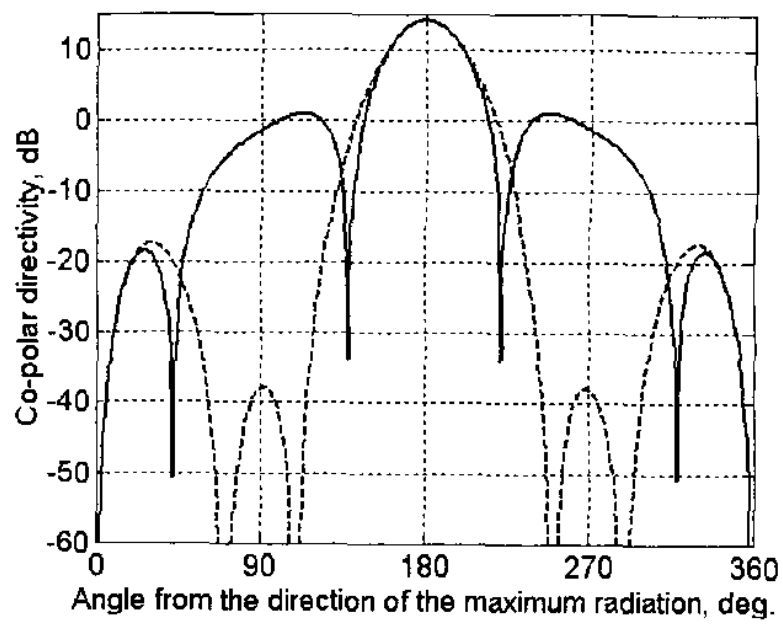

Figure 4. The co-polar directivities of the AUT model for $\phi=0^{\circ}$ and $90^{\circ}$ planes (solid line), and for the $\phi=45^{\circ}$ plane (dashed line).

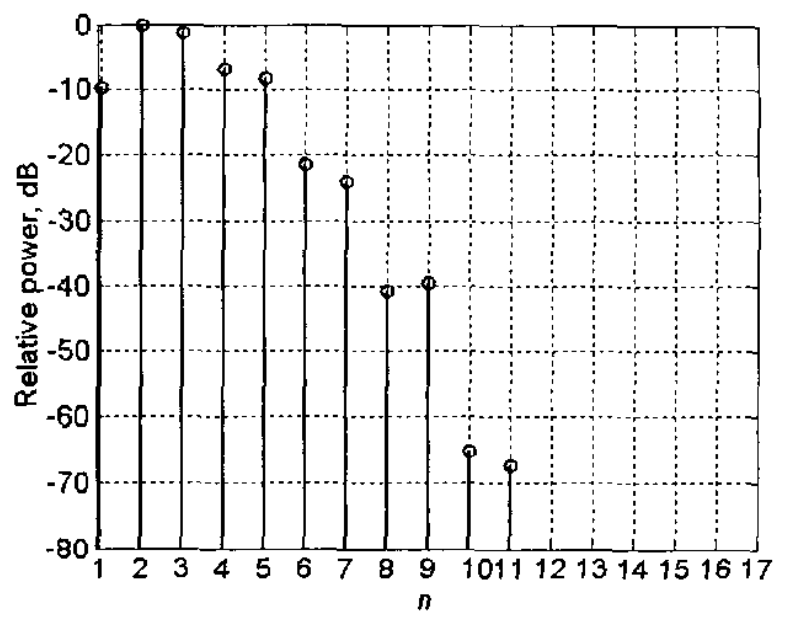

Figure 5a. The spherical n-mode power spectra of the AUT model with $d=0 \lambda$.

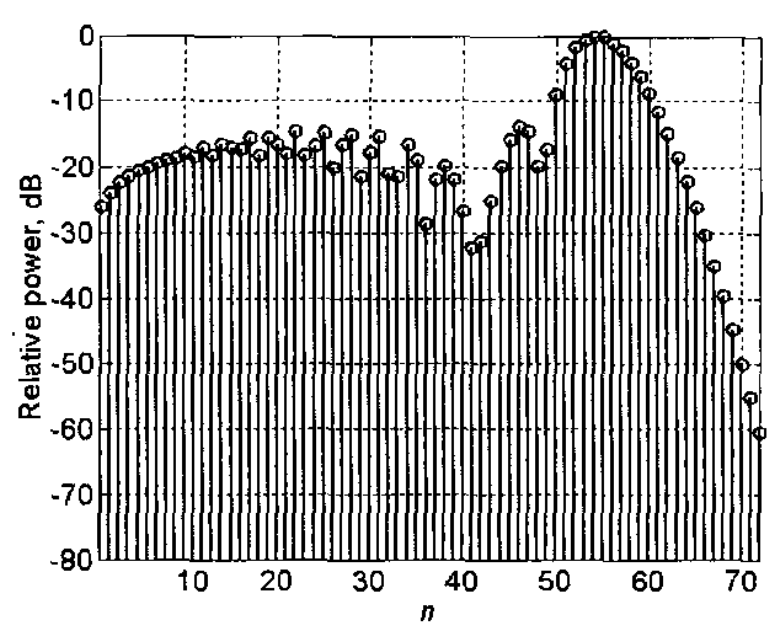

Figure 5b. The spherical $n$-mode power spectra of the AUT model with $d=9 \lambda$. 
axis distances, $d$, provided similar test cases for the purposes of validating the iterative probe-correction technique in the spherical near-field antenna measurements. This was supported by the fact that the spherical $n$-mode power spectra for the AUT model varied significantly as a function of $d$. As an example, the $n$-mode power spectra of the AUT model for $d=0 \lambda$ and $d=9 \lambda$ are shown in Figures $5 \mathrm{a}$ and $5 \mathrm{~b}$, respectively. Most of the power for the AUT model for $d=0 \lambda$ was contained in modes with $n=1, \ldots, 9$, whereas the AUT model for $d=9 \lambda$ possessed significant power in the modes with indices up to $n=70$.

\subsection{Probe Models}

A set of the probe receiving coefficients was determined from the radiation patterns of a real dual-ridged hom, measured at the DTU-ESA Spherical Near-Field Antenna Test Facility [8], for each of the frequencies $f_{1}=0.96 \mathrm{GHz}, f_{2}=1.53 \mathrm{GHz}, f_{3}=2.00 \mathrm{GHz}$, and $f_{4}=2.90 \mathrm{GHz}$. For the four frequencies, the applied truncation numbers, $v_{\max }$, were $17,13,10$, and 14 , respectively. These four

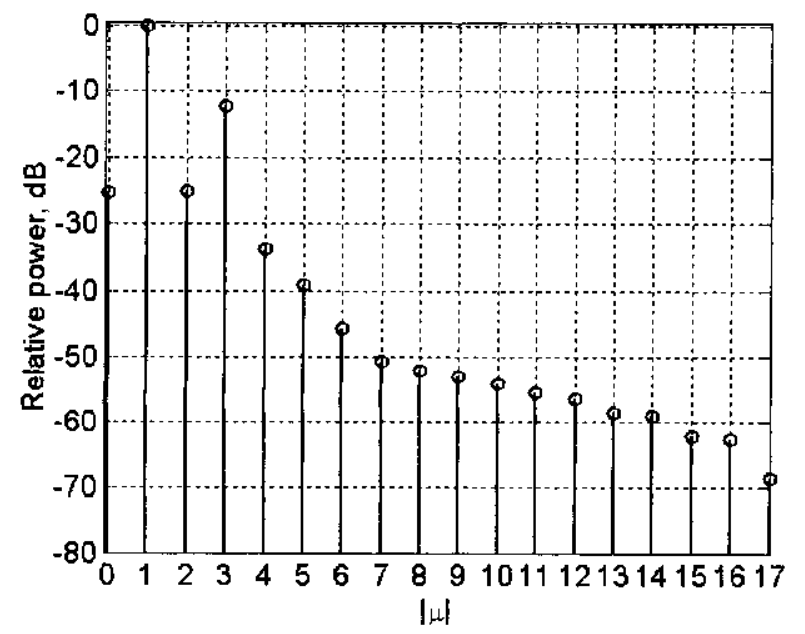

Figure $6 \mathrm{a}$. The $\mu$-mode power spectra for probel for a frequency of $0.96 \mathrm{GHz}$.

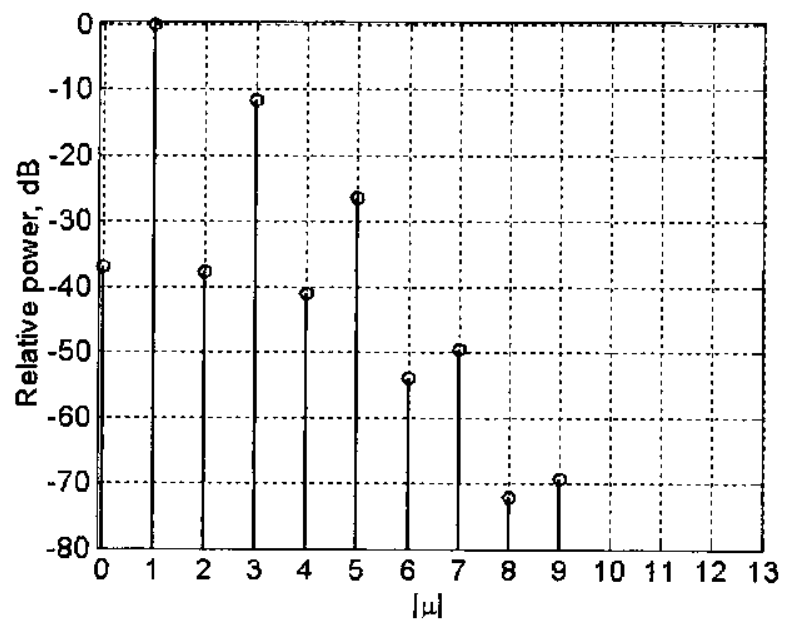

Figure 6b. The $\mu$-mode power spectra for probe2 for a frequency of $1.53 \mathrm{GHz}$.

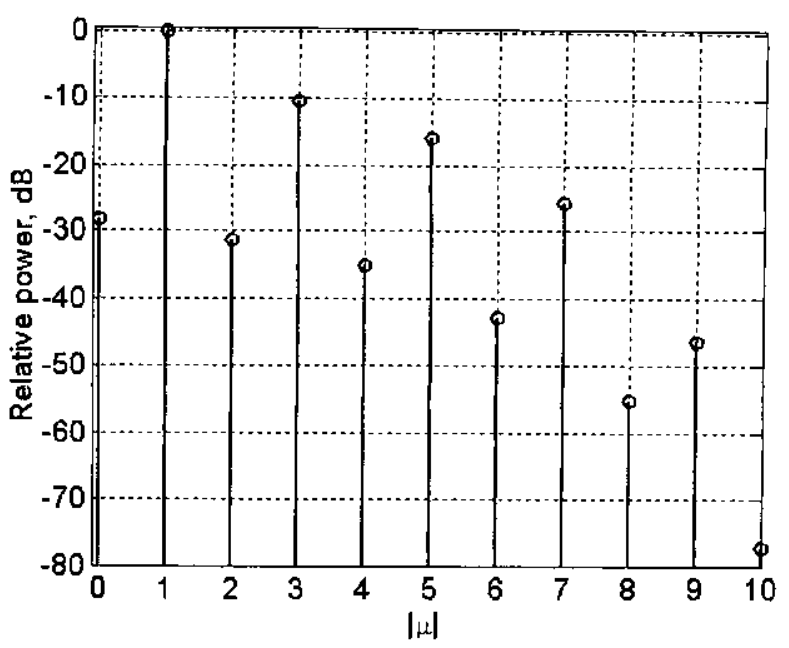

Figure $6 c$. The $\mu$-mode power spectra for probe3 for a frequency of $2.00 \mathrm{GHz}$.

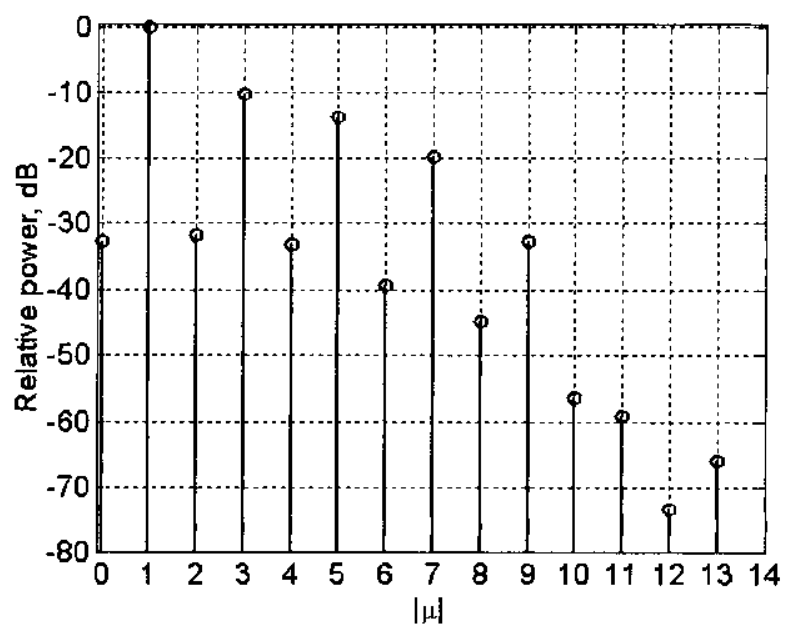

Figure 6d. The $\mu$-mode power spectra for probe4 for a frequency of $2.90 \mathrm{GHz}$.

truncated sets of probe receiving coefficients then represented the probe models, and the corresponding probe models were named probe1, probe2, probe3, and probe4. The $\mu$-mode power spectra for the probe models are presented in Figure 6.

\section{Simulations and Results}

The simulations of the spherical near-field antenna measurements were conducted for the AUT model with the four probe models with varying frequencies, measurement distances, and offaxis distances. The applied frequencies, measurement distances, and off-axis distances for a given probe model are shown in Table 1. For each simulation case, the received signal was calculated from the transmission formula of Equation (2). The iterative probe-correction technique was then applied to the calculated received signal. In most cases, just one or two iteration rounds were required to arrive at a converged result.

In Table 2, the errors in the directivity for the AUT model in angular regions $\mathrm{A}$ and $\mathrm{B}$ are given for the first-order probe correction and for the iterative probe correction with two iteration 
Table 1. The applied frequencies, measurement distances, and the ranges of applied off-axis distances for different probes.

\begin{tabular}{|c|c|c|c|}
\hline Probe & $f$ & $r$ & $\begin{array}{c}\text { Off-Axis Distance } \\
(d) \text { Range }\end{array}$ \\
\hline probel & $f_{1}$ & $18 \lambda$ & $0 \lambda-9 \lambda$ \\
\hline probe 2 & $f_{2}$ & $28 \lambda$ & $0 \lambda-15 \lambda$ \\
\hline probe 3 & $f_{3}$ & $37 \lambda$ & $0 \lambda-21 \lambda$ \\
\hline probe 4 & $f_{4}$ & $54 \lambda$ & $0 \lambda-30 \lambda$ \\
\hline
\end{tabular}

Table 2. The maximum errors in co-polar directivity [dB] in regions $A$ and $B$ for the first-order probe correction and for the iterative probe correction with two iterations.

\begin{tabular}{|c|c|c|c|c|c|c|}
\hline & \multirow[b]{2}{*}{$d(\lambda)$} & \multirow[b]{2}{*}{$r / r_{0}$} & \multicolumn{2}{|c|}{$\begin{array}{l}\text { First-Order } \\
\quad(l=0)\end{array}$} & \multicolumn{2}{|c|}{$\begin{array}{c}\text { Iterative } \\
(l=2)\end{array}$} \\
\hline & & & A & $\mathbf{B}$ & A & $\mathbf{B}$ \\
\hline \multirow{4}{*}{$\begin{array}{l}\text { probel } \\
r=18 \lambda \\
f_{1}\end{array}$} & 0 & 25.5 & 0.01 & 0.02 & 0.00 & 0.00 \\
\hline & 3 & 5.1 & 0.13 & 0.10 & 0.00 & 0.00 \\
\hline & 6 & 2.8 & 0.38 & 0.56 & 0.00 & 0.00 \\
\hline & 9 & 1.9 & 0.76 & 0.78 & 0.00 & 0.00 \\
\hline \multirow{6}{*}{$\begin{array}{l}\text { probe2 } \\
r=28 \lambda \\
f_{2}\end{array}$} & 0 & 39.6 & 0.01 & 0.02 & 0.00 & 0.00 \\
\hline & 3 & 7.9 & 0.07 & 0.09 & 0.00 & 0.00 \\
\hline & 6 & 4.3 & 0.25 & 0.18 & 0.00 & 0.00 \\
\hline & 9 & 2.9 & 0.53 & 0.41 & 0.00 & 0.00 \\
\hline & 12 & 2.2 & 0.87 & 1.09 & 0.01 & 0.01 \\
\hline & 15 & 1.8 & 1.19 & 0.66 & 0.04 & 0.03 \\
\hline \multirow{8}{*}{$\begin{array}{l}\text { probe3 } \\
r=37 \lambda \\
f_{3}\end{array}$} & 0 & 52.3 & 0.01 & 0.01 & 0.00 & 0.00 \\
\hline & 3 & 10.5 & 0.07 & 0.07 & 0.00 & 0.00 \\
\hline & 6 & 5.7 & 0.19 & 0.15 & 0.00 & 0.00 \\
\hline & 9 & 3.9 & 0.35 & 0.27 & 0.00 & 0.00 \\
\hline & 12 & 3.0 & 0.58 & 0.37 & 0.00 & 0.00 \\
\hline & 15 & 2.4 & 1.01 & 1.01 & 0.01 & 0.01 \\
\hline & 18 & 2.0 & 0.83 & 1.91 & 0.03 & 0.03 \\
\hline & 21 & 1.7 & 1.05 & 1.45 & 0.10 & 0.10 \\
\hline \multirow{6}{*}{$\begin{array}{l}\text { probe4 } \\
r=54 \lambda \\
f_{4}\end{array}$} & 0 & 76.4 & 0.01 & 0.03 & 0.00 & 0.00 \\
\hline & 6 & 8.3 & 0.14 & 0.12 & 0.00 & 0.00 \\
\hline & 12 & 4.3 & 0.46 & 0.41 & 0.00 & 0.00 \\
\hline & 18 & 2.9 & 1.04 & 0.93 & 0.03 & 0.02 \\
\hline & 24 & 2.2 & 2.45 & 1.74 & 0.14 & 0.08 \\
\hline & 30 & 1.8 & 5.47 & 4.80 & 0.48 & 0.41 \\
\hline
\end{tabular}

rounds. Region A refers to those angular directions in the $\phi=0^{\circ}$, $45^{\circ}$, and $90^{\circ}$ planes in the main beam where the co-polar directivity was greater than $1 \mathrm{dBi}$. Correspondingly, region $\mathrm{B}$ refers to those directions where the co-polar directivity was between $-5 \mathrm{dBi}$ and $1 \mathrm{dBi}$. Region $\mathrm{B}$ thus covered mainly the first sidelobes in the $\phi=0^{\circ}$ and $90^{\circ}$ planes, but also included a narrow angular region in the main beam.

The results in Table 2 show that the application of the iterative probe-correction technique with just two iterations always provided a much smaller error than the application of the firstorder probe correction, and, in most cases, the resulting error was negligible.

In some cases, e.g., for probe 4 with $d=30 \lambda$, two iterations were not sufficient to provide accurate co-polar directivity. In this particular case, the iterations were also performed further, up to $l=5$, and a maximum error in co-polar directivity of less than
$0.05 \mathrm{~dB}$ was then reached both in regions $\mathrm{A}$ and $\mathrm{B}$. It is known that the errors in the predicted radiation pattern, which are caused by the application of the first-order probe correction for non-ideal first-order probes, generally increase with a decreasing ratio between the measurement distance, $r$, and the radius of the minimum sphere of the AUT, $r_{0}$ [4]. This effect was also seen in Table 2 for every probe by following the trend of errors for the first-order correction as a function of the value of $r / r_{0}$

We now take the results for probel into closer consideration. It was seen from Figure $6 \mathrm{a}$ that the modal power of the probel with $\mu= \pm 3$ was approximately $-12 \mathrm{~dB}$ relative to the power at $\mu= \pm 1$, while the power at other modes was relatively low. For this probe, the results in Table 2 showed that the iterative probe-correction technique with just two iterations provided the co-polar directivity with negligible errors for many practical purposes in regions $\mathrm{A}$ and $\mathrm{B}$ at measurement distances $r \geq 1.9 r_{0}$.

Similarly, e.g., for probe4, the powers in modes with $\mu= \pm 3$, $\mu= \pm 5$, and $\mu= \pm 7$ were approximately $-10 \mathrm{~dB},-13 \mathrm{~dB}$, and $-20 \mathrm{~dB}$, respectively, relative to the powers in modes with $\mu= \pm 1$. Again, the power in the other modes was relatively low. For this probe, the errors in the co-polar directivity in regions $A$ and $B$ were practically negligible for $r>5 r_{0}$ as determined by the iterative probe correction with just two iterations. Increasing the number of iterations will provide more accurate results. For instance, in the case of probe4 for $r=1.8 r_{0}$, the error in the co-polar directivity in regions $\mathrm{A}$ and $\mathrm{B}$ was less than $0.05 \mathrm{~dB}$ with five iterations $(l=5)$.

Simulations were also performed to test the iterative probecorrection technique against noise, probe-pattern calibration errors, and inaccurate channel balance. These tests indicated that the iterative probe-correction technique was essentially equally sensitive to listed errors as the first-order probe-correction technique. Some of these results were reported in [9].

It is further noted that in this paper, the iterative probe correction technique was investigated mainly in the case where the number of iteration rounds was fixed at two. However, it may be necessary to apply a certain criterion to stop the iterations in some practical applications of the technique. This issue was briefly touched upon in [6].

\section{Summary and Conclusions}

An examination of the application of the iterative probe-correction technique has been reported. It has been shown by an extensive series of computer simulations for a realistic AUT model and a realistic probe model at four different frequencies that the iterative probe-correction technique can be used for probe correction, and also for other than non-ideal first-order probes in spherical near-field anterina measurements.

Some tentative guidelines on the applicability range of the iterative probe-correction technique have been given. For example, for the probe model that possessed significant power only in modes with $\mu= \pm 1$ and $\mu= \pm 3$, and for which the power in modes with $\mu= \pm 3$ was less than $-12 \mathrm{~dB}$ relative to the power in modes with $\mu= \pm 1$, the iterative probe-correction technique always worked correctly and provided accurate results at measurement distances $r>2 r_{0}$. In that case, the errors in the co-polar directivity in 
the main beam and in the first sidelobes were practically negligible with just two iterations. The $\mu$-mode spectrum of, e.g., an openended rectangular waveguide probe in its typical operating frequency fulfils the above-mentioned criteria for the modeled power [2].

The iterative probe-correction technique is important, in that it provides the possibility for a computationally efficient probe correction in spherical near-field antenna measurements for probes other than first-order probes. Once the applicability range of this technique has been clarified in detail, it could be used as a part of a spherical near-field antenna-measurement system. This could provide the possibility to build wideband spherical near-field antenna measurement systems utilizing only one probe or a few probes for the whole frequency range, together with computationally efficient data processing.

\section{References}

1. J. E. Hansen (ed.), Spherical Near-Field Antenna Measurements, London, Peter Peregrinus, Ltd, 1988.

2. T. A. Laitinen, S. Pivnenko, and O. Breinbjerg, "Aspects of Probe Correction for Odd-Order Probes in Spherical Near-Field Antenna Measurements," Antenna Measurement Techniques Association, 26th Annual Meeting \& Symposium (AMTA'04), Atlanta, GA, USA, October 17-22 2004, pp. 98-103.

3. D. Hess, "Readily Made Comparison Among the Three NearField Measurement Geometries Using a Composite Near-Field
Range," Antenna Measurement Techniques Association, 25th Annual Meeting \& Symposium (AMTA'03), Southem Califomia, USA, October 19-24 2003, pp. 486-491.

4. T. A. Laitinen, J. M. Nielsen, S. Pivnenko, and O. Breinbjerg, "Errors of First-Order Probe Correction for Higher-Order Probes in Spherical Near-Field Antenna Measurements," URSI International Symposium on Electromagnetic Theory, Pisa, Italy, May 23-27 2004, pp. 588-590.

5. T. A. Laitinen, S. Pivnenko, and O. Breinbjerg, "Comparison of Probe Correction Techniques for Spherical Near-Field Antenna Measurements," 28th ESA Antenna Workshop on Space Antenna Systems and Technologies, ESTEC, Noordwijk, The Netherlands, May 30-June 32005 , pp. 1061-1068.

6. T. Laitinen, S. Pivnenko, and O. Breinbjerg, "Iterative Ptobe Correction Technique for Spherical Near-Field Antenna Measurements," Antennas and Wireless Propagation Letters, 4, 1, 2005, pp. $221-223$.

7. A. C. Ludvig, "Definition of Cross-Polarization," IEEE Transactions on Antennas and Propagation, AP-21, 1, 1973, pp. $116-$ 119.

8. http://www.emi.dtu.dk/research/afg/snf/SNF.html.

9. T. A. Laitinen, S. Pivnenko, and O. Breinbjerg, "Sensitivities of Various Probe Correction Techniques to Noise and Inaccurate Channel Balance in Spherical Near-Fjeld Antenna Measurements," Second Intemational Conference on Electromagnetic Near-Field Characterization and Imaging (Iconic'05), Barcelona, Spain, June 8-10, 2005, pp. 411-416.

\section{Report on the AMTA Europe Symposium in Munich}

$\mathbf{T}$ The second European AMTA (Antenna Measurement Techniques Association) meeting took place in May, and was held in Munich, Germany, just before the World Cup 2006. The event attracted over 170 delegates and 18 exhibitors in total, including the main sponsors EADS Astrium GmbH and Rohde \& Schwarz. This high-level antenna symposium and exhibition was attended by delegates from the US, Canada, Asia, and many different European countries. The technical contributions were of top quality, and a balance was reached between academic and industrial content. Five students received Best Paper Awards.

The first social evening, sponsored by Nearfield Systems and EMV, took place in a traditional beer cellar in downtown Munich. On the second evening, all attendants got shot up the Olympia Tower in Munich, which is rated as the 12th-tallest structure in the world, at a staggering $290 \mathrm{~m}$.

The technical tours were hosted by our two main sponsors. The first tour took place in the Antenna Measurement Facilities of
Ottobrunn, and the second, in an active satellite ground station, just north of the Alps.

The evaluation after the event has been extremely positive. AMTA is pleased to have noticed that there is a definite need for a regular European event where the European antenna world can experience, be informed by, and network with international antenna measurement experts. On behalf of AMTA, we would like to thank all sponsors, media sponsors, and exhibitors for their trust and support. Without them, the AMTA Europe Symposium would not have been such a great success.

Margie van Esch

REALIZE GmbH

Villa Franziska, Münchner Straße 12

D-82008 Unterhaching-München, Germany

Tel:: +49-89-660799-0; Fax: +49-89-660799-66

E-mail: margie.vanesch@realize-events.de 